\title{
Supernova remnants in the Magellanic Clouds
}

\author{
Rosa N. M. Williams \\ Columbus State University, Coca-Cola Space Science Center, 701 Front Ave., \\ Columbus, GA 31901 USA \\ email: rosanina@ccssc.org
}

\begin{abstract}
At the 1998 IAU Symposium on the Magellanic Clouds, Dr. Robert Petre observed that we were reaching a time where it was possible "to study the MC SNRs at a level of detail comparable with many Galactic remnants", while retaining the benefits of a global view in the MCs. Over the past decade, many researchers have taken advantage of these newly accessible populations. New MC-wide surveys at various wavelengths have enabled broader searches for SNR candidates, extending our census of MC SNRs to less prominent objects - older SNRs, SNRs in complex regions, et cetera. The use of light-echoes has provided a new avenue to probe young SNRs. Higher spatial and spectral resolutions in many wavelength regimes have enabled detailed studies of individual remnants, revealing progenitor types, pulsar-wind nebulae, expansion details, and environmental effects.

Perhaps the newest conceptual development is the increasing use of the MC SNRs to study physical problems of wider significance to many fields of astronomy. For example, researchers have examined the energy and hot gas inputs of MC SNRs to the ISM, including their collective effects within superbubbles, in order to evaluate their effects on stellar feedback cycles in a galaxy. Other scientists have investigated the fraction of SNR energies going to the acceleration of cosmic rays, which has significant implications for the role of SNRs in cosmic-ray production. Most recently, the onslaught of Spitzer data has led to new exploration of dust in MC SNRs, allowing us to probe dust creation, depletion, and destruction in the MC SNR populations. In summary, the study of SNRs in the MCs appears to have "come of age" over the past decade, becoming a mature field with rich potential for future scientific work.
\end{abstract}

Keywords. supernova remnants, Magellanic Clouds

\section{Introduction}

A supernova can result either from catastrophic core collapse and the resulting rebound in a massive star; or from "Type Ia" explosions in which a white dwarf accretes mass from a binary companion, until it reaches the Chandrasekhar limit and detonates and/or deflagrates. In either type of supernova, once the ejecta encounter interstellar matter, a shock wave proceeds outward into the interstellar medium (ISM). This encounter creates a reflected shock through which the ejecta pass, becoming shock-heated. As these shocks expand, and sweep up more interstellar material, they create a diffuse supernova remnant (SNR). In such a remnant, the outward ("blast wave") and reflected ("reverse") shocks generate emission throughout the electromagnetic spectrum. Synchrotron radiation, which dominates at radio wavelengths, is generated by the compression of magnetic fields and the acceleration of electrons by the shock. In the post-shock cooling region we can find substantial ionic and molecular line emission, which dominates at optical, ultraviolet, and (usually) X-ray wavelengths.

A galaxy's population of supernova remnants holds key clues to the overall structure and evolution of the ISM in that galaxy. SNRs are the primary source of energy, hot 
gas, and heavy elements injected into the ISM. Both individually and collectively as superbubbles, they provide a strong influence on large-scale ISM structure in a galaxy. The energy and heavy elements provided by SNRs will influence future generations of star formation, and thus are an important factor in the dynamical evolution of galaxies. SNRs clearly play a role in the cycle of dust formation and destruction in the ISM, although the nature and extent of their contribution is still not yet fully understood.

The past decade has seen remarkable advances in the study of supernova remnants outside the Milky Way Galaxy. In particular, the Large and Small Magellanic Clouds (LMC, SMC; collectively MCs) have proven to be a fertile field for SNR studies. A generation of new instruments, on the ground and in orbit, have enabled astronomers studying MC SNRs at a wide range of wavelengths to obtain resolutions and sensitivities previously only achievable for Galactic objects. At the same time, the relatively un-obscured view of these galaxies allows studies of the SNRs in the context of their surroundings, both individually and as galaxy-wide populations.

\section{Data and demographics}

A variety of new surveys of the Magellanic Clouds have been performed in numerous wavelength regimes. These include:

- The ATCA array and Parkes telescope have been used to complete a radio survey of both the Large and Small Magellanic Cloud at 3 and $6 \mathrm{~cm}(8640$ and $4800 \mathrm{GHz})$. (See contribution by Dickel, this volume). There are also $21 \mathrm{~cm}$ maps of the LMC (Kim et al. 2003) and SMC (Stanimirović et al. 1999).

- The SAGE program (see contribution by Meixner, this volume) used Spitzer to survey the LMC and SMC in a series of narrow wavelength bands from 3 to $24 \mu \mathrm{m}$. The $\mathrm{S}^{3} \mathrm{MC}$ program also performed narrow-band infrared imaging with Spitzer (see contribution by Sandstrom, this volume, on $\mathrm{S}^{3} \mathrm{MC}$ and $\mathrm{S}^{4} \mathrm{MC}$ ). Additionally, the $A K A R I$ survey covered over half of the LMC (see contribution by Ita et al., this volume).

- The Magellanic-Clouds Emission-Line Survey covered both MCs in H $\alpha$, [S II], and [O III] (Smith et al. 2005a).

- A FUSE Legacy project resulted in a large number of ultraviolet sightlines through SNRs in the MCs.

- There has not been a systematic study of the MCs with XMM-Newton, but the pointed observations do cover a significant fraction of the Clouds.

In addition, there have been a plethora of pointed observations in all of the wavelength regimes mentioned above, which have yielded detailed images and spectral data for many MC SNRs.

- X-ray images and spectra are available via pointed observations with Chandra, XMM-Newton, ASCA, and ROSAT for almost all of the MC SNRs (e.g., Filipović et al. 2008; van der Heyden et al. 2004; Williams et al. 1999).

- Ultraviolet images and spectra are available via FUSE observations for many of the brighter SNRs (e.g., Blair et al. 2006).

- Optical images, including some Hubble Space Telescope observations, and, e.g., echelle spectra are available for most of the MC SNR sample (e.g., Payne et al. 2007).

- Infrared images, spectra, and spectral maps are available via pointed observations with Spitzer for most of the LMC SNRs and perhaps half of the SMC SNRs (e.g., Williams, Borkowski et al. 2006; Borkowski, Williams et al. 2006; Williams et al. 2006; Stanimirović et al. 2005). 


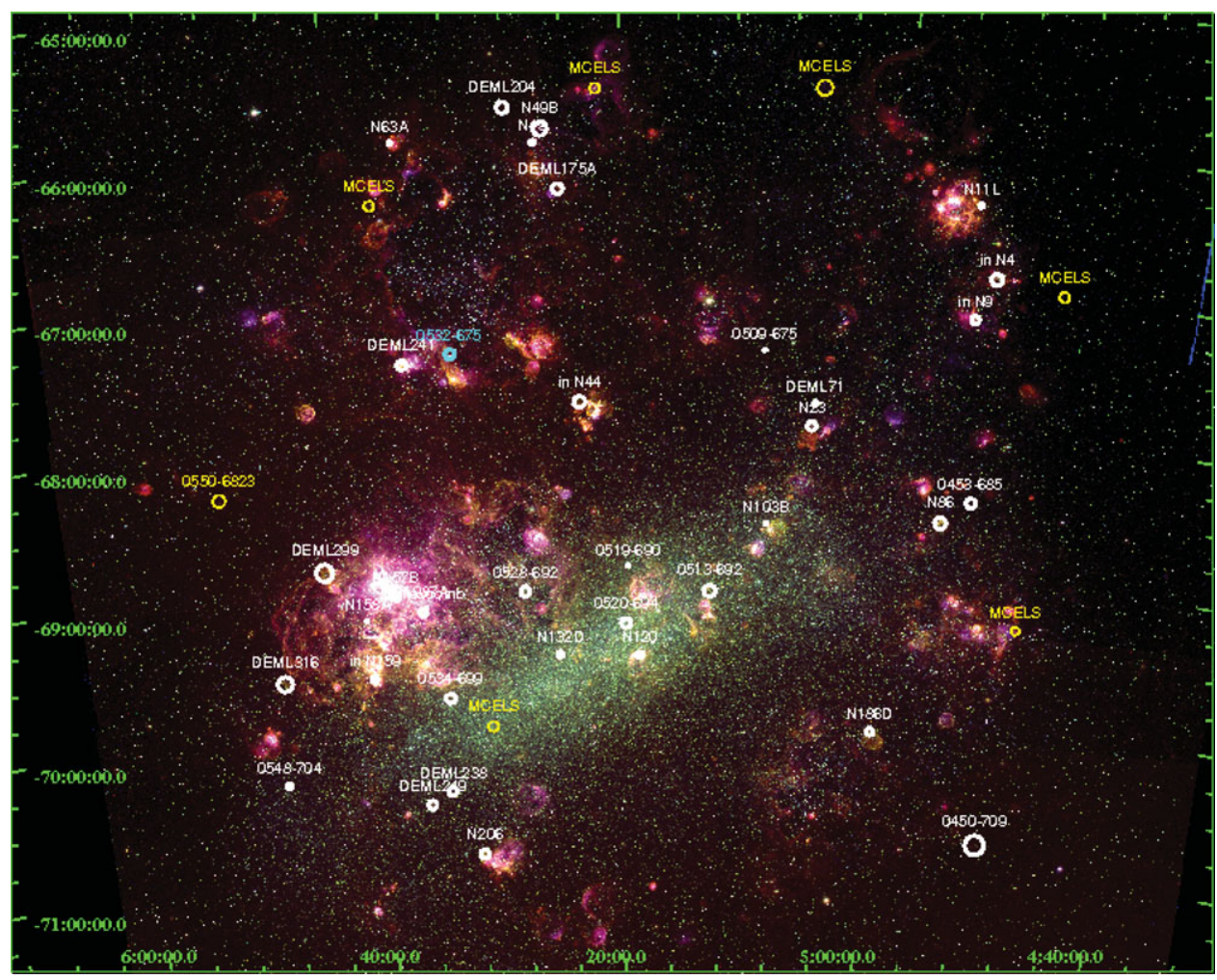

Figure 1. MCELS images of LMC with SNR known/confirmed/candidate locations overplotted. Color version: red, green, blue in image are $\mathrm{H} \alpha$, [S II], and [O III] data respectively. White SNRs are known, cyan confirmed, yellow are candidates.

Table 1. Current SNR demographics.

\begin{tabular}{|c|c|c|c|c|c|c|}
\hline & $\begin{array}{c}\text { Total } \\
\text { No. }\end{array}$ & Type Ia & $\begin{array}{l}\text { Core- } \\
\text { Collapse }\end{array}$ & $\begin{array}{c}\text { Not } \\
\text { Known }\end{array}$ & $\begin{array}{l}\mathrm{CCO} / \\
\mathrm{PWN}\end{array}$ & O-rich \\
\hline LMC & 38 & 5 & 10 & 16 & 6 & 2 \\
\hline LMC unconfirmed ${ }^{1}$ & 7 & 5 & 2 & & 1 & \\
\hline SMC & 18 & 0 & 2 & 13 & 0 & 2 \\
\hline SMC unconfirmed ${ }^{1}$ & 2 & & 4 & & 1 & \\
\hline
\end{tabular}

Notes: ${ }^{1}$ Candidate remnants, unconfirmed type, etc.

- Radio images and polarization maps are available for many of the SNRs in both MCs (e.g., Filipović et al. 2005; contribution by Filipović et al., this volume).

These new data provide a significant resource for the study of SNRs. For any given remnant, data is generally available in multiple wavelength regimes. In addition, such surveys greatly facilitate the discovery of faint, old SNRs, or SNRs in complex regions populations which are themselves of significant interest. A number of new candidate remnants have indeed been suggested over the past decade (Table 1). However, only a few have actually been confirmed as SNRs; follow-up on the list of candidates has been somewhat desultory.

From Table 1, we can see that the number of "known SNRs" in the LMC, compared to the summary table of Petre (1999) has remained constant; two of the SNRs in previous 


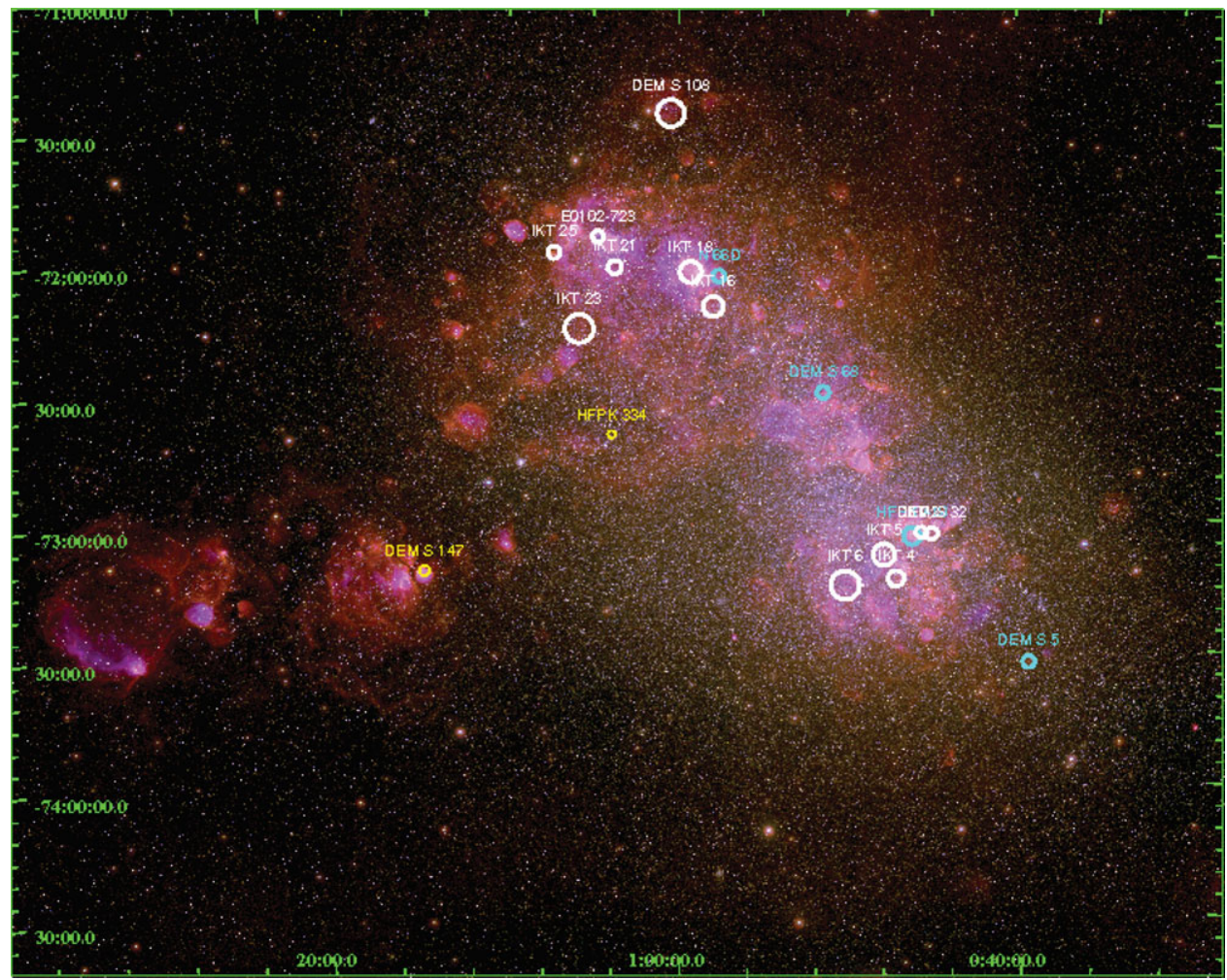

Figure 2. As Fig. 1, but now for the SMC.

list were re-identified as superbubbles, while two new discoveries joined the roster. The number of "confirmed SNRs" in the SMC has actually decreased, as a number of SNR candidates remain unconfirmed. The typing of SNRs tabulated by Petre (1999) rested partly on SNR characteristics (Balmer-line dominated SNRs typed as Ia, O-rich as CC) and partly on proximity of local OB populations (Chu \& Kennicut 1988). Current typing of supernova remnants is based primarily on SNR characteristics, including, now, typing by abundance analysis of ejecta as discussed in $\S 3.4$ below. Pulsar-wind nebulae or other compact object phenomena have been identified for six objects in the LMC (and a seventh candidate suggested), doubling the previously known numbers; none have yet been confirmed within the SMC. These objects are discussed in $\S 3.2$.

\section{Individual SNR studies}

The wealth of new data, and of high-resolution, high-sensitivity instruments, has led to an explosion of research on Magellanic Cloud SNRs in the past decade. It is beyond the scope of this work to cover each paper in detail; instead, I summarize a few notable developments. These include recent developments in the study of SN 1987A; progress in uncovering and studying pulsar-wind nebulae (PWN) and other compact-object phenomena; observations of light-echoes from the youngest remnants; the use of X-ray spectra to determine abundances, allowing comparisons with model predictions; and observations of SNR interactions with their surroundings.

\subsection{SNR 1987A}

The most studied SNR in the MCs is of course SN/SNR 1987A; a brief search of the literature turns up as many papers about this object as for the rest of the MC SNRs 
together. A full review of this object is beyond the scope of this work; I will here only touch on a very select set of recent findings.

In 1995, the first "hot spot" was observed as an unresolved brightening along the equatorial ring surrounding SN 1987A. By 1999, there were many such spots, signaling the beginning of the transition from a supernova to a supernova remnant (Lawrence et al. 2000) as supernova ejecta encountered the circumstellar ring. Since then, emission interpreted as being from ejecta heated by the reverse shock has been observed (Michael et al. 2003; McCray 2007). The time-evolution of this SNR is being studied on a continuing basis at a wide range of wavelengths, with new observations every few months. This allows ongoing study of time-dependent effects in the evolution of a very young SNR. (e.g., Park et al. 2006; Heng et al. 2006; Smith et al. 2005b; Utrobin \& Chugai 2005; Manchester et al. 2005).

The physical issues examined in SNR 1987A are many and varied. These include an inventory of the nucleosynthesis products, (e.g., Heng et al. 2008), and examinations of specific isotopes, (e.g., ${ }^{55} \mathrm{Fe}$, Leising 2006). Exhaustive study has been made of shock structure, ionization stages, and internal shock velocities (Dewey et al. 2008; Gröningsson et al. 2008; Heng \& McCray 2007; Reighard \& Drake 2007; Haberl et al. 2006). Dust formation and destruction in SNRs is a subject of high current interest, and is studied in depth in SN 1987A (Dwek et al. 2008; Ercolano et al. 2007; Bouchet et al. 2006); the general case will be discussed in $§ 4.3$. Although efforts have also been bent toward finding a pulsar, the search has not yet resulted in a detection; see Manchester (2007) for a review. One should note that the upper limits for a detection are still relatively generous, so these non-detections are unsurprising.

\subsection{Compact-object phenomena}

The search for compact object phenomena in other SNRs has been more fruitful. Three examples had been known before the start of this decade. SNR 0540-693 in the LMC hosts the well-known pulsar (PSR) 0540-69, as well as a PWN bright in optical and X-rays. Morse et al. (2006) used Hubble Space Telescope data to show that the outer rim of the PWN had a "skin" of [O III] emission; Petre et al. (2007) followed up on this in a detailed study of the X-ray structure of the PWN, among other things showing that the $\mathrm{X}$-ray extent was bounded by this [O III] feature.

In the SNR N157B, a long-suspected pulsar was detected by Marshall et al. (1998). Shortly afterward, Wang \& Gotthelf (1998) identified X-ray emission from a PWN surrounding this pulsar. Further study of this region by Wang et al. (2001) showed it to have a "comet-like" head and tail structure, which they interpreted as a bow shock from the pulsar's supersonic motion through the SNR ejecta. (It should be noted that a variant interpretation has been given by van der Swaluw (2004), who argues that the motion of the pulsar is subsonic and that it is interacting with the reverse shock in the SNR.)

A soft gamma-ray repeater (SGR) $0526-66$ is co-located on the sky with supernova remnant N49 in the LMC. However, whether the two objects were physically associated was considered questionable for some time (e.g., Kaplan et al. 2001). However, studies by Kulkarni et al. (2003) and Park et al. (2003) identified an X-ray counterpart to the SGR. Kulkarni et al. (2003) were able to use this counterpart to estimate the presumptive pulsar's spin-down rate, and calculate its age; that age is reasonably close to the estimated age of N49, which does suggest an association between the two.

In this decade, we have more than doubled the numbers of confirmed or probable compact-object phenomena. (Note that this also adds to the number of SNRs we can confidently classify as resulting from core-collapse SNe.) Gaensler et al. (2003) identified a small-diameter radio and X-ray source within SNR 0453-685, and determined it to be a relatively "Vela-like" PWN, probably subsonic. Klinger et al. (2002) noted a "peculiar 
linear feature" in radio maps of N206-SNR, and a subsequent X-ray examination by Williams et al. (2005) confirmed the presence of another "cometary" PWN resulting from a bow shock. Bamba et al. (2006) have more tentatively identified what may be a similar X-ray structure within the SNR DEM L 241. Finally, Hughes et al. (2006) and Hayato et al. (2006) uncovered and studied a point-like source in the SNR N23, whose properties are consistent with those of an object similar to the compact central object (CCO) recently discovered in the Galactic remnant Cas A.

\subsection{Light echoes}

It has long been known that the light of a supernova, as it propagates through the ISM, may be reflected at a delay toward us from various ISM features in a phenomenon known as a "light-echo". Such light-echoes had been noted to occur with SN 1987A (Crotts 1988; Rosa et al. 1988) in the Large Magellanic Cloud. In the current decade, however, the SuperMACHO collaboration discovered three more light-echoes within the LMC (Rest et al. 2005). These light-echoes were traced back to three of the LMC's youngest SNRs: N103B, SNR 0509-675, and SNR 0519-690. From the rate of travel, they were able to precisely determine ages for these SNRs; later studies of shock velocities from ultraviolet observations (Ghavamian et al. 2007) provided an independent check on these estimates. As the light from these echoes is a reflection of the supernova itself, Rest et al. (2005) were able to identify the progenitor type for each of the SNRs as Type Ia events; this is consistent with the Balmer-line dominated emission for which these remnants have been known (Tuohy et al. 1982; Smith et al. 1991). In addition, these light-echoes have the potential to be sensitive probes of the ISM through which they pass (e.g., Chevalier 1986; Crotts 1988).

\subsection{Spectral analysis of abundances}

Hughes et al. (1995) compared the X-ray spectra of some of the youngest, ejecta-dominated LMC SNRs to nucleosynthesis models for Type Ia versus core-collapse supernovae. The authors were able to distinguish between Type Ia and core-collapse progenitors for all of the SNRs in their sample. Later researchers found that in many cases, even when SNRs are dominated by swept-up ISM, there are still enough ejecta signatures in their X-ray spectra to identify the progenitor types with reasonable confidence. By this method, a number of older MC SNRs have recently been identified as resulting from Type Ia supernovae (e.g., Hendrick et al. 2003; Hughes et al. 2003; Lewis et al. 2003; Borkowski et al. 2006). In some instances, SNRs from core-collapse events have also been identified (e.g., Park et al 2003b,c; Williams et al. 2008).

An example of the application of this technique was provided by the two remnants of DEM L 316 in the LMC. The system has two shells which overlap along the line of sight. Williams et al. (1997) used various features of these remnants to argue that the two were coeval and actually colliding. However, Nishiuchi et al. (2001) used ASCA X-ray data to show that the abundances in one shell were most probably consistent with a Type Ia origin. Following up on this, Williams \& Chu (2005) used Chandra X-ray spectra to confirm that result, and further identified the other shell as probably of core-collapse origin. The hypothesis that the two shells were coeval was clearly rendered improbable by these findings!

The increasing identification of remnants as Type Ia SNRs also provides a sample for more precise comparisons of SNR abundances with models. For example, Warren \& Hughes (2004) used SNR 0509-675 to test nucleosynthesis models of Type Ia supernovae, and found that a delayed-detonation model best fit the observed abundances. Addressing a different issue, Badenes et al. (2007) investigated a number of known Type Ia SNRs to 
test a particular model in which accretion winds from the binary system of the progenitor white dwarf excavate a cavity in the surrounding ISM. However, the authors concluded that this "wind" model was inconsistent with the observed properties of ejecta in their sample of MC SNRs.

\subsection{SNR interactions with surroundings}

Another feature of the last decade has been the increase in studies examining MC SNRs in the context of their surroundings, and their interactions with the nearby ISM. The MCs offer rich samples, which have enabled research to study SNRs interacting with molecular clouds (Bilikova et al. 2007; Koo et al. 2007; Warren et al. 2003); with H II regions and complexes (Reid et al. 2006; Gorjian et al. 2004; Danforth et al. 2003; Nazé et al. 2002; Dickel et al. 2001; Williams et al. 2008); with (and within) superbubbles (Chen et al. 2006; Townsley et al. 2006); and even, in one case, with a single stellarwind bubble (Velázquez et al. 2003 ). These studies address such questions as how the interaction with the denser material with a molecular cloud influences postshock cooling in an SNR; observational features of a superbubble that point to a recent interior SNR; the evolution of an SNR in the rarified environment of a superbubble; the formation and evolution of superbubbles themselves; and similar issues.

\section{Using SNRs to address astrophysical issues}

Possibly the most significant development in MC SNR research is the increasing use of Magellanic Cloud SNRs to address broader questions of interest to astronomy. Three examples of such work are discussed below: the impact of SNRs on the global ISM; the SNR role in cosmic-ray acceleration; and the creation and destruction of dust in SNRs.

\subsection{SNR inputs to global ISM}

SNRs are a key element of the stellar feedback cycle that is discussed extensively in these proceedings. Remnants affect the ISM individually, driving local conditions; and collectively as they combine with stellar wind to form the large-scale $(>100 \mathrm{pc})$ superbubbles. Thus, SNRs provide much of the energy that drives the stellar feedback processes in the ISM: thermal energy as hot gas; kinetic energy which goes to mechanical feedback (see contributions by Chu and by Oliveira, this volume); and nonthermal energy, some of which may go to cosmic-ray acceleration. Of course, SNRs also are the source of the nucleosynthesis mentioned above, providing heavy elements to the ISM.

A persistent question has been the "filling factor" of the hot gas phase (in the standard three-phase model of McKee \& Ostriker (1977) for the ISM) in the Magellanic Clouds. In order to pin down this elusive quantity, two things are required; a fairly complete census of the sources of hot gas, i.e. SNRs and superbubbles; and knowledge of how long hot gas persists in SNR and superbubble interiors. The first element is addressed by uncovering faint, old remnants, and by trying to account for the number of SNRs within the rarefied cavities of superbubbles. The second element is addressed by studying the oldest SNRs; recent work has shown that hot gas persists deep in SNR interiors to late stages. In fact, these hot reservoirs are thought to result in "mixed-morphology" remnants, in which we see shell-like radio structure but X-ray filled interiors. Again, this line of inquiry must also account for the heating of gas inside superbubbles by SNR shocks.

\subsection{Cosmic-ray acceleration}

Energy from SNRs can go to another component: they are thought to be a primary source of $\mathrm{GeV}$ and $\mathrm{TeV}$ cosmic rays. Hughes et al. (2000) examined the SMC SNR E0102-723. 
Based on the observed shock velocities, they inferred a high postshock ion temperature. Ions and electrons exchange energy through the Coulomb process, which links the electron temperature to the ion temperature. In this case, the electron temperature measured from $\mathrm{X}$-ray spectra was considerably lower than expected from the shock velocity. The authors suggested that the "missing" energy may have largely gone to cosmic-ray acceleration.

Another question of interest is the maximum energy to which SNRs can accelerate cosmic rays. It is known that the nonthermal spectrum in SNRs steepens significantly from the extrapolated spectrum of radio synchrotron emission. Hendrick \& Reynolds (2001) modeled the change in slope from radio to X-ray spectra, and fit this for 11 Magellanic Cloud SNRs. The "cutoff" in their model gives the maximum energy for cosmic-ray acceleration, and their fits indicated maximum cosmic-ray energies of about $80 \mathrm{TeV}$. While this is consistent with the overall expectation for the energy range SNRproduced cosmic rays, it shows that SNRs are unlikely to propel cosmic rays over the "knee" in the cosmic-ray spectrum above $100 \mathrm{TeV}$.

\subsection{Dust production and destruction}

Supernova ejecta are thought to be a major source of dust in the ISM, along with other primary sources such as the winds of evolved stars. Indeed, emission signatures of dust have been observed in supernovae. Warm $(100 \mathrm{~K})$ dust is expected to radiate strongly in infrared, but has proven difficult to detect in any but the youngest SNRs (e.g., Reach et al. 2006).

In observing SNR 1987A, Dwek et al. (2008) concluded that the infrared emission was primarily from swept-up ISM dust, not from SNR ejecta. Further, the dust-to-gas mass ratio in SNR 1987A has decreased over time - a sign of ongoing grain destruction. Tappe et al. (2006) also found infrared emission from dust in the young SNR N132D, but also inferred that the observed dust was from the swept-up ISM. They found a dust-to-gas mass ratio of about of tenth of the average ratio for the LMC. Notably, the authors found that the spectrum of the infrared emission was consistent with grain losses from dust destruction by sputtering. Borkowski, Williams et al. (2006) and Williams, Borkowski, et al. (2006), for LMC samples of Type Ia and core-collapse SNRs, respectively, likewise interpreted their Spitzer observations of infrared emission as being from swept-up dust. They, too, found low dust-to-gas mass ratios.

In addition, Williams et al. (2006) published a preliminary study, later confirmed by spectral mapping data, that showed that for certain specific remnants, ionic and molecular line emission from post-shocked gas forms a substantial fraction of the infrared emission. This leads to ever-decreasing dust estimates: not all SNRs are detected in infrared; when detected, some of the infrared emission may be line emission from the gas component; when dust is found it is often thought to be dust swept up from the ISM, rather than newly formed in ejecta; and even of the swept-up dust, often less is found in the SNR than is found in the typical MC ISM! SNRs may prove to be net destroyers of dust, rather than producers of it.

However, there is still room for substantial dust contribution from SNRs. An example is provided by SNR E0102-723. When this SNR was studied by Stanimirović et al. (2005), they estimated the proportion of line emission contribution to the infrared, and noted that the dust continuum appeared to originate from regions of the SNR associated with the reverse shock as well as the outer blast wave. This suggests that some emission is indeed from dust in the newly shock-heated ejecta. Sandstrom (see contribution by Sandstrom et al., this volume) has been analyzing spectra from this SNR, and finds strong evidence for actual ejecta-formed dust. While there is still less dust found than models predict 
should be produced by SNRs, one must also consider that much of this dust may remain within the unshocked ejecta, and this "cold" dust may not be readily observable.

\section{Summary}

SNR studies in the Magellanic Clouds can now be considered a mature field of research. Researchers continue, of course, with the categorization, characterization, and comparison of Magellanic SNRs with Galactic SNRs. Work has been pushed to ever more sophisticated analyses of SNRs themselves, with MC SNRs being used to test various models of SNR origins, structure, and evolution. In addition, current studies are using MC SNRs to address key physical problems significant to a broad range of topics within astronomy.

\section{Acknowledgements}

The author gratefully acknowledges NASA Long-Term Space Astrophysics grant NNG05GC97G, which has supported the author's research in this subject as well as travel to this conference.

\section{References}

Badenes, C., Hughes, J. P., Bravo, E., \& Langer, N. 2007, ApJ, 662, 472

Bamba, A., Ueno, M., Nakajima, H., Mori, K., \& Koyama, K. 2006, A\&A, 450, 585

Bilikova, J., Williams, R. N. M., Chu, Y. -H., Gruendl, R. A., \& Lundgren, B. F. 2007, AJ, 134, 2308

Blair, W. P., Ghavamian, P., Sankrit, R., \& Danforth, C. W. 2006, ApJS, 165, 480

Borkowski, K. J., Hendrick, S. P., \& Reynolds, S. P. 2006, ApJ, 652, 1259

Borkowski, K. J., Williams, B. J., et al. 2006, ApJ, 642, L141

Bouchet, P., Dwek, E., Danziger, J., et al. 2006, ApJ, 650, 212

Chen, Y., Wang, Q. D., Gotthelf, E. V., Jiang, B., Chu, Y. -H., \& Gruendl, R. 2006, ApJ, 651, 237

Chevalier, R. A. 1986, ApJ, 308, 225

Chu, Y. -H. \& Kennicutt, R. C., Jr. 1988, AJ, 96, 1874

Crotts, A. 1988, ApJ, 333, L51

Danforth, C. W., Sankrit, R., Blair, W. P., Howk, J. C., \& Chu, Y. -H. 2003, ApJ, 586, 1179

Dewey, D., Zhekov, S. A., McCray, R., \& Canizares, C. R. 2008, ApJ, 676, L131

Dickel, J. R., Williams, R. M., Carter, L. M., Milne, D. K., Petre, R., \& Amy, S. W. 2001, AJ, 122,849

Dwek, E., et al. 2008, ApJ, 676, 1029

Ercolano, B., Barlow, M. J., \& Sugerman, B. E. K. 2007, MNRAS, 375, 753

Filipović, M. D., Haberl, F., Winkler, P. F., et al. 2008, A\&广A, 485, 63

Filipović, M. D., Payne, J. L., Reid, W., Danforth, C. W., Staveley-Smith, L., Jones, P. A., \& White, G. L. 2005, MNRAS, 364, 217

Gaensler, B. M., Hendrick, S. P., Reynolds, S. P., \& Borkowski, K.J. 2003, ApJ, 594, L111

Ghavamian, P., Blair, W. P., Sankrit, R., Raymond, J. C., \& Hughes, J. P. 2007, ApJ, 664, 304

Gorjian, V., Werner, M. W., Mould, J. R., et al. 2004, ApJS, 154, 275

Gröningsson, P., Fransson, C., Lundqvist, P., et al. 2008, A\&A, 479, 761

Haberl, F., Geppert, U., Aschenbach, B., \& Hasinger, G. 2006, A\& A, 460, 811

Hayato, A., Bamba, A., Tamagawa, T., \& Kawabata, K. 2006, ApJ, 653, 280

Hendrick, S. P., Borkowski, K. J., \& Reynolds, S. P. 2003, ApJ, 593, 370

Hendrick, S. P. \& Reynolds, S. P. 2001, ApJ, 559, 903

Heng, K. \& McCray, R. 2007, ApJ, 654, 923

Heng, K., Haberl, F., Aschenbach, B., \& Hasinger, G. 2008, ApJ, 676, 361

Heng, K., McCray, R., Zhekov, S. A., et al. 2006, ApJ, 644, 959 
Hughes, J. P., Rafelski, M., Warren, J. S., Rakowski, C., Slane, P., Burrows, D., \& Nousek, J. 2006, ApJ, 645, L117

Hughes, J. P., Ghavamian, P., Rakowski, C. E., \& Slane, P. O. 2003, ApJ, 582, L95

Hughes, J. P., Rakowski, C. E., \& Decourchelle, A. 2000, ApJ, 543, L61

Hughes, J. P., Hayashi, I., Helfand, D., et al. 1995, ApJ, 444, L81

Kaplan, D. L., Kulkarni, S. R., van Kerkwijk, M. H., Rothschild, R. E., Lingenfelter, R. L., Marsden, D., Danner, R., \& Murakami, T. 2001, ApJ, 556, 399

Kim, S., Staveley-Smith, L., Dopita, M. A., Sault, R. J., Freeman, K. C., Lee, Y., \& Chu, Y. H. 2003, ApJS, 148, 473

Klinger, R. J., Dickel, J. R., Fields, B. D., \& Milne, D. K. 2002, AJ, 124, 2135

Koo, B. -C., Lee, H. -G., Moon, D. -S., et al. 2007, PASJ, 59, 455

Kulkarni, S. R., Kaplan, D. L., Marshall, H. L., Frail, D. A., Murakami, T., \& Yonetoku, D. 2003, ApJ, 585, 948

Lawrence, S. S., Sugerman, B. E., Bouchet, P., et al. 2000, ApJ, 537, L123

Leising, M. D. 2006, ApJ, 651, 1019

Lewis, K. T., Burrows, D. N., Hughes, J. P., Slane, P. O., Garmire, G. P., \& Nousek, J. A. 2003, ApJ, 582, 770

Manchester, R. N. 2007, in S. Immler, K. Weiler, \& R. McCray (eds.), Supernova 1987A: 20 Years After, AIP Conf.Proc. 937 (New York: AIP), p. 134

Manchester, R. N., Gaensler, B. M., Staveley-Smith, L., Kesteven, M. J., \& Tzioumis, A. K. 2005, ApJ, 628, L131

Marshall, F. E., Gotthelf, E. V., Zhang, W., Middleditch, J., \& Wang, Q. D. 1998, ApJ, 499, L179

McCray, R. 2007, in S. Immler, K. Weiler, \& R. McCray (eds.), Supernova 1987A: 20 Years After, AIP Conf.Proc. 937 (New York: AIP), p. 3

McKee, C. F. \& Ostriker, J. P. 1977, ApJ, 218, 148

Michael, E., McCray, R., Chevalier, R., et al. 2003, ApJ, 593, 809

Morse, J. A., Smith, N., Blair, W. P., Kirshner, R. P., Winkler, P. F., \& Hughes, J. P. 2006, ApJ, 644, 188

Nazé, Y., Hartwell, J. M., Stevens, I. R., Corcoran, M. F., Chu, Y. -H., Koenigsberger, G., Moffat, A. F. J., \& Niemela, V. S. 2002, ApJ, 580, 225

Nishiuchi, M., Yokogawa, J., Koyama, K., \& Hughes, J. P. 2001, PASJ, 53, 99

Park, S., Zhekov, S. A., Burrows, D. N., Garmire, G. P., Racusin, J. L., \& McCray, R. 2006, ApJ, 646, 1001

Park, S., Burrows, D. N., Garmire, G. P., Nousek, J. A., Hughes, J. P., \& Williams, R. M. 2003a, ApJ, 586, 210

Park, S., Hughes, J. P., Slane, P. O., Burrows, D. N., Warren, J. S., Garmire, G. P., \& Nousek, J. A. 2003b, ApJ, 592, L41

Park, S., Hughes, J. P., Burrows, D. N., Slane, P. O., Nousek, J. A., \& Garmire, G. P. 2003c, ApJ, 598, L95

Payne, J. L., White, G. L., Filipović, M. D., \& Pannuti, T. G. 2007, MNRAS, 376, 1793

Petre, R., Hwang, U., Holt, S. S., Safi-Harb, S., \& Williams, R. M. 2007, ApJ, 662, 988

Petre, R. 1999, in Y. H. Chu, N. Suntzeff, J. Hesser, \& D. Bohlender (eds.), New Views of the Magellanic Clouds, IAU Conf.Proc. 190 (ASP), p. 74

Reach, W. T., Rho, J., Tappe, A., et al. 2006, AJ, 131, 1479

Reid, W. A., Payne, J. L., Filipović, M. D., Danforth, C. W., Jones, P. A., White, G. L., \& Staveley-Smith, L. 2006, MNRAS, 367, 1379

Reighard, A. B. \& Drake, R. P. 2007, Ap\&SSS, 307, 121

Rest, A., Suntzeff, N. B., Olsen, K., et al. 2005, Nature, 438, 1132

Rosa, M., Gouiffes, C., Ruiz, M., \& Beresford, A. C. 1988, IAU Circular, 4564, 1

Smith, R. C., Points, S. D., Chu, Y. -H., Winkler, P. F., Aguilera, C., Leiton, R., \& MCELS Team 2005a, BAAS, 37, 1200

Smith, N., Zhekov, S. A., Heng, K., McCray, R., Morse, J. A., \& Gladders, M. 2005b, ApJ, 635, L41

Smith, R. C., Kirshner, R. P., Blair, W. P., \& Winkler, P. F. 1991, ApJ, 375, 652 
Stanimirović, S., Staveley-Smith, L., Dickey, J. M., Sault, R. J., \& Snowden, S. L. 1999, MNRAS, 302,417

Stanimirović, S., Bolatto, A. D., Sandstrom, K., Leroy, A. K., Simon, J. D., Gaensler, B. M., Shah, R. Y., \& Jackson, J. M. 2005, ApJ, 632, L103

Tappe, A., Rho, J., \& Reach, W. T. 2006, ApJ, 653, 267

Townsley, L. K., Broos, P. S., Feigelson, E. D., Brandl, B. R., Chu, Y. -H., Garmire, G. P., \& Pavlov, G. G. 2006, AJ, 131, 2140

Tuohy, I. R., Dopita, M. A., Mathewson, D. S., Long, K. S., \& Helfand, D. J. 1982, ApJ, 261, 473

Utrobin, V. P. \& Chugai, N. N. 2005, A\&BA, 441, 271

van der Heyden, K. J., Bleeker, J. A. M., \& Kaastra, J. S. 2004, A\&\&A, 421, 1031

van der Swaluw, E. 2004, Adv. Sp. Res., 33, 475

Velázquez, P. F., Koenigsberger, G., \& Raga, A. C. 2003, ApJ, 584, 284

Wang, Q. D. \& Gotthelf, E. V. 1998, ApJ, 494, 623

Wang, Q. D., Gotthelf, E. V., Chu, Y. -H., \& Dickel, J. R. 2001, ApJ, 559, 275

Warren, J. S. \& Hughes, J. P. 2004, ApJ, 608, 261

Warren, J. S., Hughes, J. P., \& Slane, P. O. 2003, ApJ, 583, 260

Williams, B. J., Borkowski, K. J., et al. 2006, ApJ, 652, L33

Williams, R. M. \& Chu, Y. -H. 2005, ApJ, 635, 1077

Williams, R. M., Chu, Y. -H., Dickel, J. R., Gruendl, R. A., Seward, F. D., Guerrero, M. A., \& Hobbs, G. 2005, ApJ, 628, 704

Williams, R. M., et al. 2008, ApJ, submitted

Williams, R. M., Chu, Y. -H., \& Gruendl, R. 2006, AJ, 132, 1877

Williams, R. M., Chu, Y. -H., Dickel, J. R., Gruendl, R. A., Seward, F. D., Guerrero, M. A., \& Hobbs, G. 2005, ApJ, 628, 704

Williams, R. M., Chu, Y. -H., Dickel, J. R., Petre, R., Smith, R. C., \& Tavarez, M. 1999, ApJS, 123,467

Williams, R. M., Chu, Y. -H., Dickel, J. R., Beyer, R., Petre, R., Smith, R. C., \& Milne, D. K. 1997, ApJ, 480, 618 